its speedy cure, all seem to indicate that it was not of that nature, and that its cause must be looked for in one of two circum. stances, or in a combination of both.

1st. The opposing surfaces of peritoneum being united by inflammation at the point of exit from the abdomen, and the sac external to this sloughing; it may be that the ulcerative process tending to throw off this slough, extended to or slightly beyond the point of union between the gut and sac, and so opened the former; and if so, the cause of the opening, practically, was the death of the sac.

2ndly. It may be that a stricture, which is not enongh to cause death of the whole gut included, may yet be effectual to produce ulceration of the part to which it is applied, or rather of a part of the circumference to which it is applied. It seems possible that one point may be so firmly constricted as to have the circulation stopped in it, although the constriction may not be so tight all round as to stop the circulation in all the gut beyond. This may have been the case here, aud so the opening may have been caused, or it is possible that the two causes might act together.

If I am correct in my judgment of the opening and its cause, we are taught by it,

1st. To bear in mind, in operating, that we must not, beyond what is absolutely necessary, disturb the newly-acquired connections of the sac, as, by doing so, we must further diminish its vitality, which is already lowered by its separation from its natural connections, and by the constriction to which it has been subjected.

2ndly. It points out the propriety of withdrawing so much of the intestine, previous to reducing it, as to bring into view the constricted point, provided this can be done by traction so gentle as not to destroy any union that may have taken place in consequence of inflammation at the point of exit; for it is evident that had $I$ in this case withdrawn the gut, I might have seen nothing to warn me of the risk of returning it, and yet the ulceration might have taken place within the abdominal cavity, and the result in all probability would have been fatal.

\section{The following Case}

I do not know the history of during life, but simply that she died, and after death there was found a large ventral hernia below and on each side of the umbilicus. The woman was upwards of fifty years of age, but how long the tumour had existed is unknown. On opening the sac it was found to consist of two distinct ravities; one on each side of the linea alba, communicating by an opening, apparently formed in this fibrous structure, which formed the septum between them. We thus had as it were two herniæ, each having its distinct sac, and each its point of stricture separate from the other.
In operating upon large herhiz, and espe. cially ventral or umbilical herniæ, it is advisable, for obvious reasons, to avoid an extensive opening of the sac, and to content ourselves with an incision upon its neck; and in such cases, too, the proper course to pursue, not unfrequently, is to relieve the stricture and not to return the contents of the sac into the abdominal cavity. Had this case during life called for operation, and this rule been adopted, its true nature might never have been known, and the true stricture might never have been suspected to exist. An isolated case does not show the impropriety of the rule, but enforces the propriety of an accurate examination into the history of the outward form of such hernix, and as far as possible into their internal structure and contents.

Haddington, Dec، 10, 1842.

\section{CASE OF}

\section{ABSCESS IN THE BRAIN.}

By G. Pyemont Smith, M.D., Leeds.

THE following case seems to me to possess some interest both in a physiological and a pathological point of view :-

George Moorhouse, a bricklayer, aged 38, came to me on Wednesdas, Nov. 9th, complaining of headach, which he had had occasionally for six weeks, especially in an evening, after his work. On examination I found the pulse quite natural, abont 80 ; no heat of head or throbbing of the temples, and the bowels rather inclined to costiveness. A common aperient was ordered, and $\mathbf{I}$ saw nothing more of him till Monday, the 14th Nov., when I visited him, and found him labouring under violent tic donloureux of the left side of the face, the pain was intermittent, and most violent at night; the pulse and tongue in a natural state. I ordered an embrocation of ammonia water, tinctnre of opium, and spirits of wine, to be rubbed on the face during the paroxysm, and a pul. gative to regulate the bowels.

15. The pain of face is relieved by the embrocation, but returned again after a time; bowels freely open. Continue embrocation.

17. I was sent for about five o'clock in the evening to see my patient, as he had had a fit. On inquiry $I$ found he had been sitting in his chair about two o'clock, resting his head on his left hand, when he hadbeen seized with a sudden peculiar sensation of thrilling and numbness on the right side of his face, and right arm. He had likewise found himself unable to speak. He got up, took a draught of water, and walked about the room for a few minutes till these sensations left him. He recovered the use of his speech in about a quarter of an hour. At about half-past four he had a second similar attack, and had not recovered from its effects when I saw him. He was then unable to 
articulate more than " no" or " yes." When the tongue was protruded it pointed to the right side, and the right half of the tongue appeared swollen, the left half being natural. The patient was quite collected, and had been so during both the fits (if they may be so called). The pulse was about 80 , and natural. R Croton oil, gtt. iij ; bread crumbs, gr. $x v$. Mix. Divide into three pills. Take one every fourth hour.-B Iodide of potassium, Эij ; syrup of puppies, $3 \mathrm{ij}$; water, zviij. Mix. Take one ounce every fourth hour.

18. He gradually recovered his speech last evening, and can now talk nearly as well as usual. The tongue is still protruded to the right side. Slight paralysis of the right side of the face; the right eyelid does not close so completely as the left. The croton oil has produced several copious evacuations. There has been no pain of the face or head since the first fit. Continue the mixture and the iodide of potassium.

19. Much the same; pulse 82 .

21. Had another fit last night, and has not recovered his speech, but replies to any questions that are put to him by writing on a slate. The paralysis of the right side of the face appears to be increasing; he grasps equally well with the right as with the left hand; tongue rather white. Continue the croton oil and the mixture of iodide of potassium. Apply ablister to the neck.

22. The blister has risen well. symptoms are much the same.

23. He is now no longer able to write, although the grasp of the right hand is nearly as strong as that of the left; other symptoms continue much the same.

24. On this day, at the request of a brother of the patient, Mr. Pawson saw him with me. All the symptoms continue much the same. The right hand has lost a little more of its power. There is no pain or uneasiness of any kind in the head; pulse about 80 ; tongue rather white; bowels re. gular. Continue the mixture, and apply two blisters behind the ears.

25. The blisters have risen well; other symptoms are much the same; the patient for the first time has remained in bed all day.

27. At the request of the patient's friends Dr. Hobson saw him with me to-day. The paralysis of the right arm and hand had increased considerably, the hand being quite powerless; pulse 84, natural; other symptoms much the same. The head was ordered to be shaved, and a blister applied to the left half.-R Sirychnia, gr. $\frac{1}{8}$; blue pill, gr. ij; comp. extr. of colocynth, gr. iij. Mix. Make a pill, to be taken every sixth hour. Continue the mixture of iodide of potassium.

28 . Found the patient in a state of somnolence, in which he had remained since three o'clock the preceding afternoon, but had risen twice during the night to void urine. Various means were tried to arouse him, such as striking the face and hands with a wet towel, but although these means were ineffectual, he wiped the wet off his face with his left hand, and if his bedclothes were thrown off, he attempted to cover himself again. He had taken three of the pills, the first having been taken at about seven o'clock on the preceding evening. The blister had risen very little. The pills were ordered to be discontinued. The eyes, when opened, appeared slightly dilated, and there was some heat of the forehead. Apply evaporating lotion to the forehead, and continue the mixture.

29. The coma is now complete; he has voided urine, twice, in bed; he no longer takes his medicine. There is occasional hiccough. Apply equal parts of strong mercurial ointment, and spermaceti cerate to the blistered half of the head, and a blister to the other half. An enema, with salt, was also ordered.

30. The blister has not risen; the breathing has become stertorous, and the pulse weak and wiry. Death took place at about half-past twelve, a.m.

\section{Post-mortem Examination Forty-five Hours after Death.}

The bones of the cranium were rather thin ; the membranes of the brain healthy, the arachnoid containing an ordinary quantity of serum, of a slightly red colour; the left hemisphere of the cerebrum softer than the right; the base of the brain and cerebellum presented a healthy appearance. At about the centre of the left hemisphere of the cerebrum there was found a cavity, rather larger than a walnut, containing greenish-coloured pus, with an offensive smell, the parietes of the cavity presenting the appearance of the commencement of the formation of a cyst.

Leeđs, Dec. 10, 1842.

\section{SUETTE MILIARE.}

Five of the departments of France, in different parts of the kingdom, were visited during the past year with this pestiferous disease, the sweating-sickness of English authors, which caused such ravages in England in the fourteenth and fifteenth centuries, but which is now happily banished from this country, and is even rare among our neighbours. In one part of the department Dordogne, out of a population of 82,200 persons, 10,400 were attacked by the disease, of whom 800 , or one in thirteen, perished. It was observed to rage mostly in marshy situations, and to be best treated in a manner similar to intermittent fever, viz., with quinine, and other tonics, taking particular care to interfere in no way with the march of the miliary eruption. - Gazette des Hopitaux. 